\title{
Study of Different Burst Scheduling Algorithms Using FDLs as QoS in Wavelength Division Multiplexing OBS Networks
}

\author{
L. Netak ${ }^{1}$ and G. Chowdhary ${ }^{2}$ \\ ${ }^{1}$ Dept. of Computer Engineering, Dr. B. A. T. U., Lonere-402 103, Raigad, (MS), India \\ ${ }^{2}$ School of Computational Sciences, SRTMU, Nanded, (MS), India \\ \{ldnetak@dbatu.ac.in, girish.chowdhary@gmail.com\}
}

\begin{abstract}
Optical burst switching (OBS) is envisioned to be one of the most promising technology to support all-optical switching in the optical Internet. OBS is finding potential in bandwidth hungry applications like, grid computing, video conferencing, video telephony and video on demand. In this critical high speed Internet based applications, where bursts losses should be minimized. In OBS based networks, there exists the possibility that bursts may contend with one another at intermediate nodes. Typically, contention resolution in IP networks is implemented by storing excess packets in electronic buffer (RAM). However, electronic like buffer is unavailable and optical buffer is constructed from fiber delay lines (FDL). An FDL is simply the length of fiber and offer a deterministic delay. We present simulation results which show the effect of the use of FDLs of various delays to reduce bursts losses. The simulation results show that such limited optical buffers can significantly reduces burst losses in the network.
\end{abstract}

\section{Introduction}

The ever-increasing demand for higher bandwidth due to growth of bandwidth intensive applications such as audio, video-on-demand, video conferencing, grid computing, online trading, and other multimedia applications motivated to search alternatives for traditional electronic networks. Wavelength division multiplexing (WDM) is one such technology developed to handle the future bandwidth demands. Several approaches have been proposed to take advantages of optical communication and in particular switching techniques in WDM networks. Commonly used switching techniques are, optical circuit switching (OCS), optical packet switching (OPS), and optical burst switching (OBS)[1][2]. Out of these switching techniques, OBS is a promising and feasible way for the next-generation optical Internet before OPS realized. The benefit of OBS over conventional OCS is that there is no need to dedicate a wavelength for the entire session between a pair of nodes. Moreover, OBS is more viable than OPS as the data does not need to be buffered or processed at the intermediate nodes. An OBS based WDM networks has three types of nodes, namely an ingress, egress, and a network of core nodes. At the ingress node, various type of client data from the access networks are aggregated into a data burst (DB). A DB is logical aggregation of Internet protocol (IP) packets and it is transmitted entirely in the optical domain. To avoid buffering and processing of the optical DB at the intermediate nodes, a burst header packet (BHP) prepared and sent ahead of DB. BHP contains DB control information such as, length, arrival time, and quality of service (QoS), if required. BHPs are transmitted on a dedicated control channel, while DBs transmitted on separate data channels.

The time gap between a BHP and the corresponding data burst send is called offset time. Also, it is, ensured that, offset time sufficient enough to enable the processing of BHP and to configure the switches at the core nodes. Initially the offset time is set to $\mathrm{T}$, and calculated $\mathrm{h} \delta+\mathrm{ts}$, where $\mathrm{h}$ is the number of hops, $\delta$ is processing time required on each hop and ts is the average time required to configure switches along the path. When a BHP arrive at intermediate nodes, scheduling algorithm is used to schedule DB with the help of control information.

Once a DB reaches to egress node, it is disassembled into packets which each one routed through the access network. The main objective of scheduling algorithm is to reduce bursts losses and improve throughput of the networks. One of the key issue in OBS networks is burst scheduling algorithm. An ideal scheduling algorithm should be able to process BHP before corresponding DB arrives and to find out a best suitable outgoing channel for DB transmission. If the reservation fails before DB arrival, then the DB will be dropped. The various scheduling algorithms have been proposed to schedule a burst, such as latest available unused channel (LAUC), LAUC with void filling (VF). LAUC is a simple, easy to implement and widely used algorithm. LAUC uses horizon (unused time of channel after which no burst allocation) to schedule burst on wavelength channels. Whereas, LAUC-VF uses voids, and it is nothing but the time gap between two successive scheduled bursts on same channel.

B. Iyer, S. Nalbalwar and R.Pawade (Eds.)

ICCASP/ICMMD-2016. Advances in Intelligent Systems Research.

Vol. 137, Pp. 697-705.

(C) 2017- The authors. Published by Atlantis Press

This is an open access article under the CC BY-NC license (http://creativecommons.org/licens)es/by-nc/4). 
There are several variants of LAUC-VF algorithms, such as minimum starting void (Min-SV) and minimum ending void (Min-EV). In this paper, we have studied the performance of different scheduling algorithms with and without FDLs, and single stage feed-back FDL architecture is used. The detailed study has been carried out by using simulation, and found that channel utilization is improved. The rest of the paper is organized as follows. In the next section, we analyzed different scheduling algorithms. In section III detail outline of QoS improvement through FDL pool. In section IV simulation results and performance is analyzed based on the burst losses. Finally, conclusion is given in section V.

\section{Burst Scheduling Algorithms}

When a BHP is processed at the core node, a burst scheduling algorithm is used to schedule an incoming burst. Since the scheduling is done an online fashion based on the arrival time of the burst, efficient scheduling algorithms are necessary to minimize burst losses. The main objective of scheduling algorithm should be to minimize the voids and burst losses. A void on wavelength is the unused time between two consecutive bursts. Several scheduling algorithms have been proposed, in which main objective is to minimize burst losses. The following are the existing burst scheduling algorithms used in OBS network.

\section{- Latest available unused channel with void filling (LAUC-VF) algorithm [11], [12]:}

The void filling version of the LAUC scheduling algorithm, keeps track of all the voids on the wavelengths and tries to schedule burst in one of the void whichever is minimum among all voids. The LAUC-VF scheduling algorithm is more complex to implement than the LAUC scheduling algorithm because it has to maintain additional state information for each wavelength which is the size of the available voids. Since the LAUC-VF scheduling algorithm can use the voids created by previously scheduled bursts. The link utilization is higher than that in case of the LAUC scheduling algorithm. However, the LAUC-VF scheduling algorithm takes longer time to schedule burst compared to the LAUC scheduling algorithm.

- Best-Fit (BF) algorithm [11]: In this algorithm, a scheduler keeps track of void for each channel. It also maintains start time and end time of voids for each data channels. The scheduler tries to search for a void such that newly created void is the smallest void before and after scheduled burst.

- Minimum starting void (Min-SV) algorithm [11], [12]: In this algorithm, a scheduler keeps track of void for each channel. It also maintains start and end time of voids for each data channels. Scheduler tries to search for a void such that newly created voids is the smallest voids after scheduled bursts.

- Minimum Ending Void (Min-EV) algorithm [11], [12]: In this algorithm, a scheduler keeps track of void for each channel. It also maintains start and end time of voids for each data channel. The scheduler tries to search for a void such that newly created void is the smallest void before the scheduled burst.

BF, Min-SV and Min-EV algorithms are the variants of LAUC-VF algorithm. All the void filling algorithms yield better bandwidth utilization and burst loss rate than LAUC algorithm. But all the scheduling algorithm has a longer execution time than LAUC algorithm. Fig. 1 show, how the different scheduling algorithm behaves when a new burst arrives at the node. Table I shows the comparisons of different scheduling algorithm [11]. Further, summarizes the above

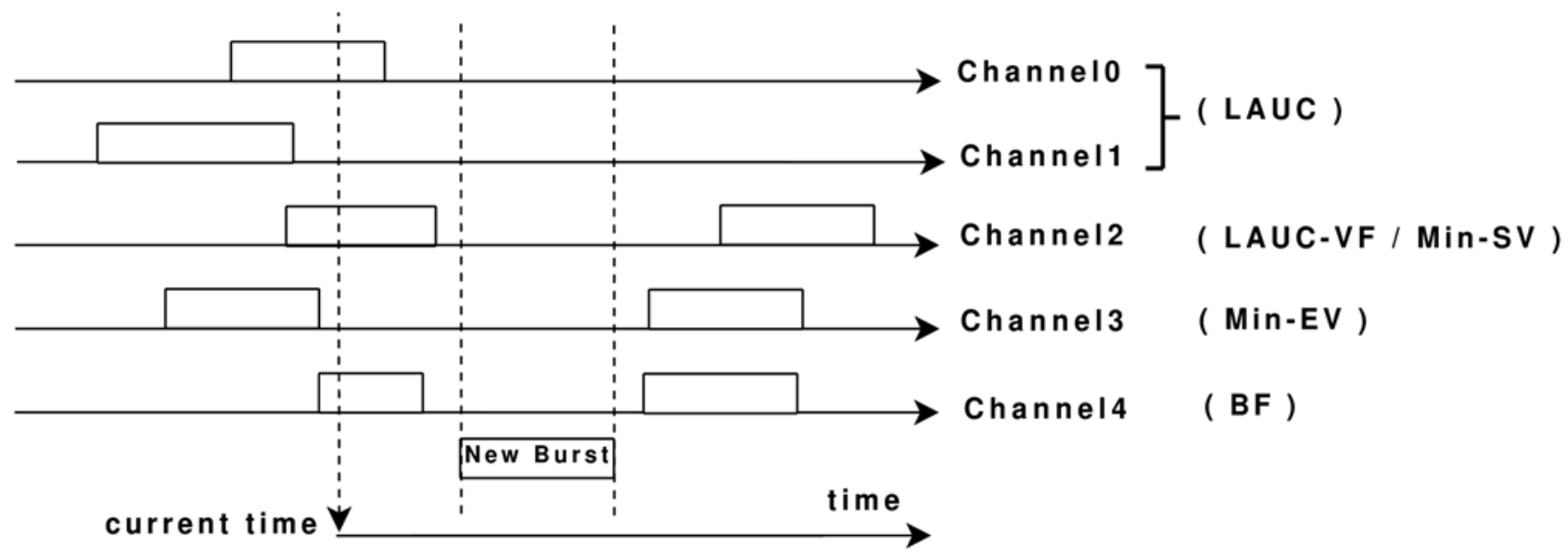

Fig. 1. An example showing how a new burst is scheduled by using different scheduling algorithms. 
- Latest available unused channel (LAUC) algorithm [11], [12]:

It is very simple and widely used, also it is called Horizon. The algorithm maintains the latest available unscheduled time on each wavelength and when a new burst arrive, it is scheduled on a wavelength such that the void size is minimized. Simplicity and ease of implementation are the main advantages of the LAUC scheduling algorithm as the scheduler needs to remember only one value for each data wavelength which is the unscheduled time. But the LAUC scheduling algorithm cannot utilize the voids created by the previously scheduled bursts because it looks only the latest unscheduled time. This causes excessive burst losses when the variation in the offset time is large.

Table I Comparison of Different Scheduling Algorithms

\begin{tabular}{|c|c|c|c|}
\hline Scheduling Algorithms & Time Complexity & $\begin{array}{c}\text { State } \\
\text { Information }\end{array}$ & $\begin{array}{c}\text { Bandwidth } \\
\text { Utilization }\end{array}$ \\
\hline LAUC & $\mathrm{O}(\mathrm{W})$ & Horizon $_{i}$ & Low \\
\hline LAUC-VF & $\mathrm{O}(\mathrm{W} \log \mathrm{m})$ & $\mathrm{S}_{\mathrm{i}, \mathrm{j}}, \mathrm{E}_{\mathrm{i}, \mathrm{j}}$ & High \\
\hline BF & $\mathrm{O}(\mathrm{W} \log \mathrm{m})$ & $\mathrm{S}_{\mathrm{i}, \mathrm{j}}, \mathrm{E}_{\mathrm{i}, \mathrm{j}}$ & High \\
\hline Min-SV & $\mathrm{O}(\log \mathrm{m})$ & $\mathrm{S}_{\mathrm{i}, \mathrm{j}}, \mathrm{E}_{\mathrm{i}, \mathrm{j}}$ & High \\
\hline Min-EV & $\mathrm{O}(\log \mathrm{m})$ & $\mathrm{S}_{\mathrm{i}, \mathrm{j}}, \mathrm{E}_{\mathrm{i}, \mathrm{j}}$ & High \\
\hline
\end{tabular}

- W : Number of wavelengths at each output port.

- $\mathrm{m}$ : Maximum number of data bursts on all channels.

- Horizon $_{\mathrm{i}}$ : Horizon of the I th data channel.

- $\quad \mathrm{S}_{\mathrm{i}, \mathrm{j}}$ and $\mathrm{E}_{\mathrm{i}, \mathrm{j}}$ : Starting and ending time of $j$ reservation on channel $i$.

\section{QoS Improvement Mechanism through Single Stage Feed-Forward Optical Buffering}

In OBS, contention will occur if multiple bursts from different input ports are destined for the same output port at the same time [7]. Typically, contention resolution in traditional electronic packet switching networks is implemented by storing excess packet in Random Access Memory (RAM) buffers.

However, RAM-like optical buffers are yet unavailable. Currently, optical buffers are constructed from fiber delay lines (FDLs). An FDL is simply length of fiber and hence offer fixed delay. Once a burst has entered, it must emerge after a fixed length of time later. It is impossible to either remove the burst from the FDL earlier or hold it for a longer time.

The fundamental difficulty facing the designer of an optical burst switch is to implement variable-length buffers from these fixed-length FDLs. Optical buffering also known as limited buffering and may be categorized either single-stage, which have only one block of parallel delay lines, or multi-stage, which have several blocks of delay lines, cascaded together. Single stage optical buffers are easier to control, but multistage implementation may lead to more saving on the amount of hardware used [7].

\section{A. QoS Improvement Mechanisms in OBS}

There are two basic models for QoS: relative QoS and absolute QoS. In the relative QoS model, the performance of each class is not defined qualitatively in absolute terms. Instead, the QoS of one class is defined relative of other classes. For example, the burst of high priority is guaranteed to experience lower loss probability than the burst of lower priority. However, the loss probability of a high-priority traffic still depends on the traffic load of lower priority traffic; and no upper bound on the loss probability is guaranteed for the highpriority traffic. The absolute QoS model provides a worst-case QoS guarantee to applications. This kind of hard guarantee is essential to support applications with delay and bandwidth constraints. Moreover, from a service provider's point of view, the absolute QoS model is preferred in order to ensure that each user receives an expected level of performance. An efficient admission control and resource provisioning mechanisms are needed to support the absolute QoS model. 


\section{B. Contention Resolution and QoS}

As OBS networks provide connection-less transport, the bursts may contend with one another at the intermediate nodes. Burst losses due to contention are a key concern in OBS networks. Contention among two bursts occurs due to their overlap in time when they arrive simultaneously on two different links or wavelengths and request the same outgoing wavelength. In electronic packet switching networks, contention is handled by buffering. However, in optical networks, buffers are difficult to implement and there is no optical equivalent of random access memory.

Contention resolution schemes may be used to provide QoS in an all-optical core network. Some of the main techniques used to resolve contention for a wavelength at the core nodes are mentioned below and also discussed optical buffing in details.

Wavelength Conversion: If two bursts contend for the same wavelength at a core node, they can be sent different wavelengths resolve the contention. Wavelength conversion is the process of converting the bursts on one wavelength in an incoming link to a different wavelength in the outgoing link. This helps to increase the wavelength reuse and the wavelengths can be spatially reused to carry different bursts on different fiber links in the network.

Deflection Routing: This is a technique of deflecting the bursts onto alternate paths to the destination in case of contention for a wavelength at a core node. Deflection routing has many disadvantages. It is by nature sub-optimal, since it only considers the congestion of the

current switch, not the state of links in the deflected path. Deflection routing implementation requires changing the offset time of a burst which is impossible without the use of buffers. Since deflection routing is done by each individual node without cooperation from the rest of the network, it can lead to routing loops.

Burst Segmentation: In burst segmentation, a portion of the burst which overlaps with another burst is segmented instead of dropping the entire burst. When two bursts contend for the same wavelength, either the head of the contending burst or the tail of the other burst is segmented and dropped. Therefore segmentation can be classified into head dropping or tail dropping. The remaining segment of the burst is transmitted successfully to the destination, thereby increasing the packet delivery ratio.

Optical Buffering (Limited Buffering): Optical buffering also known as limited buffering. Current optical buffers can be classified as either single-stage, or having only one block of parallel delay line, or multi-stage, which have several blocks of delay lines, cascaded together. Single stage optical buffers are easier to control, but multi-stage implementation may lead to more saving on the amount of hardware used [7]. Optical buffers can also be classified as having feed-forward feedback configuration. The following Fig. 2, shows single-stage optical buffer architecture.

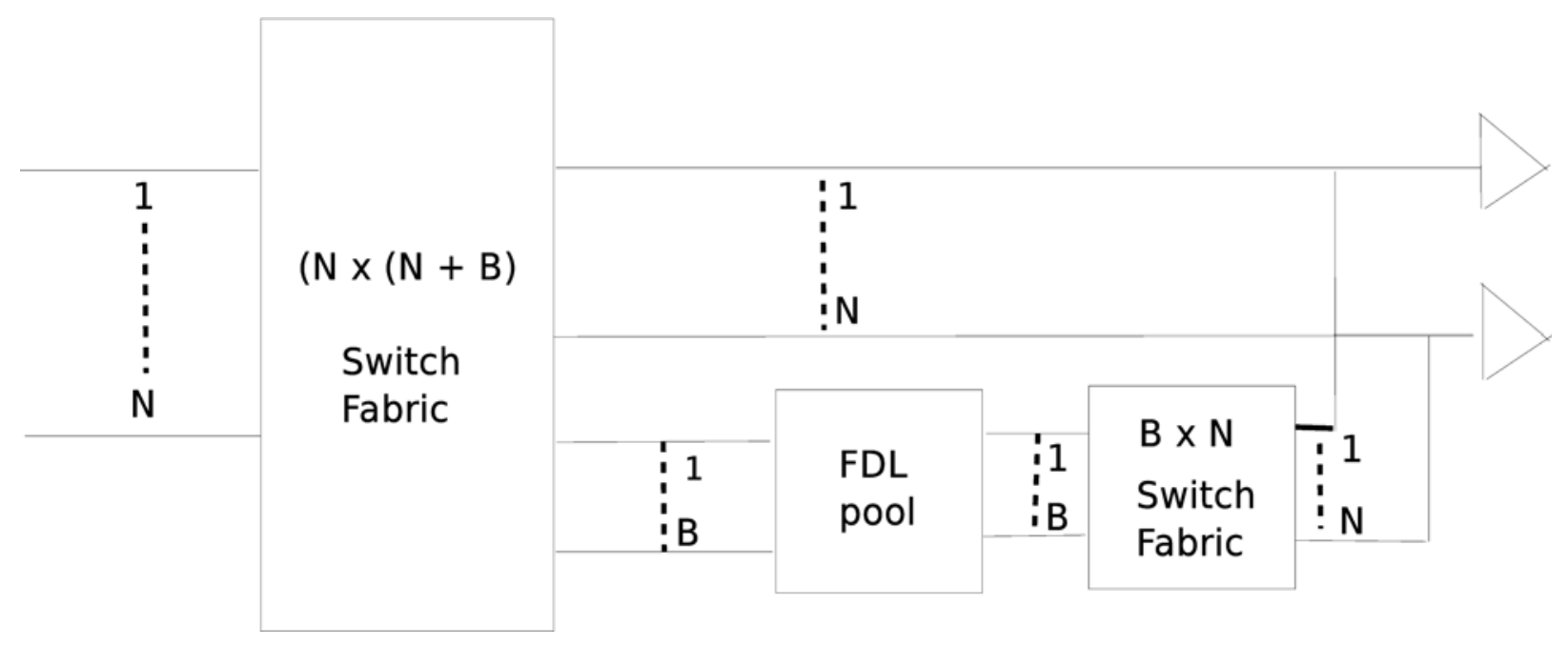

Fig. 2. Single-stage feed-forward optical buffer architecture ([1]). 
In a feed-forward configuration, delay lines connect the output of a switching stage of input of the next switching stage. In a feedback configuration, delay lines connect the output of switching stage back to the input of the same stage. Long holding time and certain degrees of variable delay can be easily implemented with the feedback configuration by varying the number of loops a burst undergoes. However, each loop causes some loss in single power. Therefore, a burst cannot be stored indefinitely in feedback architecture.

In a feed-forward configuration, delay lines with different lengths must be used to achieve variable delays. This architecture attenuates all signals almost equally because every burst passes through the same number of switches. Hybrid combination of feed-forward and feedback architectures is also possible [7]. Based on the position of buffers, packet switches fall into one of three major categories: input, output buffering and shared buffers. In input-buffered switches, a set of buffers is assigned for each input port. This configuration has poor Performance due to the head-of-line blocking problem.

Consequently, it is never proposed for purely optical implementation. In output-buffered switches, a set of buffers is assigned to each output port. Most optical switches emulate output buffering since the delay in each output optical buffer can be determined before the burst enters it. Shared buffering is similar to output buffering except that all output ports share a common pool of buffers.

Due to their hardware-saving characteristics, multi-stage and/or shared-buffer architectures are predominant in optical switching proposals. Fig. 2, shows two single-stage, shared-buffer switch architecture [14] with feedforward configuration where $\mathrm{N}$ and $\mathrm{B}$ are the number of input ports and the number of FDLs, respectively. They both contain FDL pool that is shared among all output ports. In the feed-forward configuration, burst delayed only once, whereas the feedback configuration allows them to be delayed multiple times. Since the FDLs are optical fibers themselves, it is possible for them to hold multiple bursts of different wavelengths simultaneously. However, this comes at the expense of increased complexity in scheduling algorithms. Compare to single-stage buffer architecture, multi-stage counterpart are much more complex. They contain several primitive switching elements, connected together by FDLs, usually in a feed-forward configuration. Multi-stage buffers can achieve buffer depth of several thousands. A delay of $1 \mathrm{~ms}$ requires over $200 \mathrm{Km}$ of fiber.

Due to limitations of buffers, optical buffering alone as a means of contention resolution may not be effective under high load or busty traffic conditions. In addition to buffering bursts optically, it is also possible to buffer bursts electronically. Electronic buffering can be accomplished by sending the bursts up to the electronic switching or routing layer. The disadvantage of of such an approach is that the network loses transparency, and each node must have electronic switching or routing capabilities, resulting in higher network costs and also requiring electronic memories which must keep up with the speeds of optical

Networks. Furthermore, a greater load will be placed on the processing capabilities of the electronic switch or router. An alternative would be to implement electronic buffers directly as a part of the optical switch itself. In this case each node will require additional transmitters and receivers, and would need to be aware of the transmission format of the bursts; however no additional electronic routing or switching capability would be required.

\section{Simulation Results}

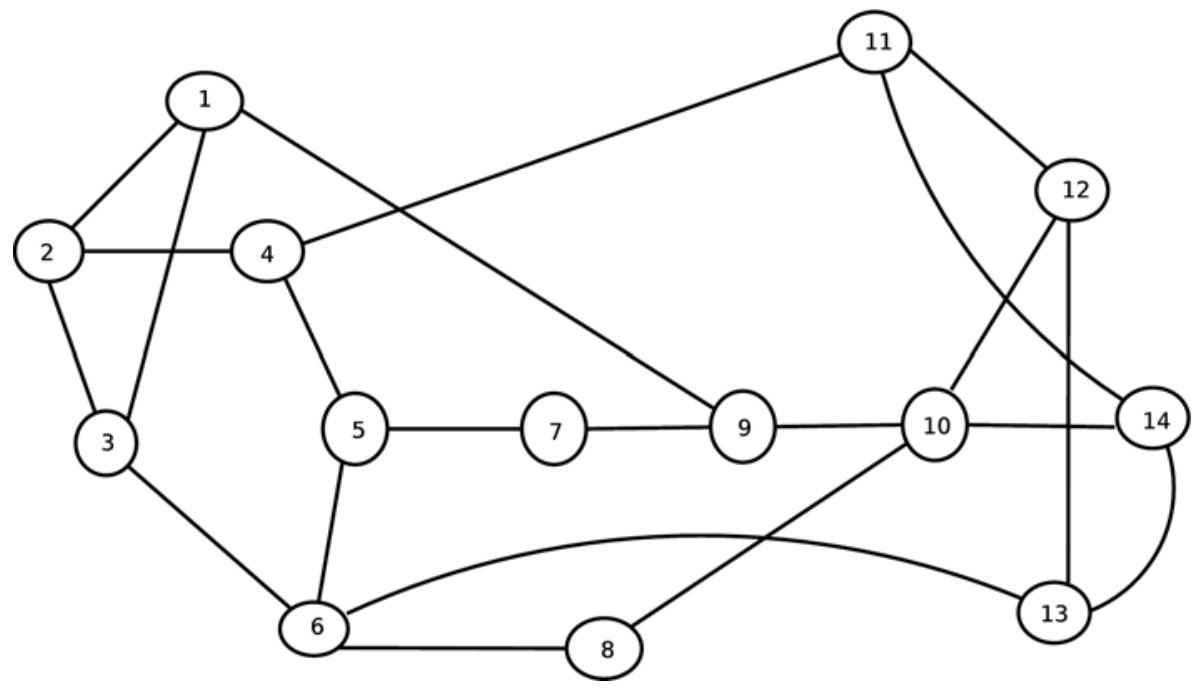

Fig. 3. Simulation network topology model. 


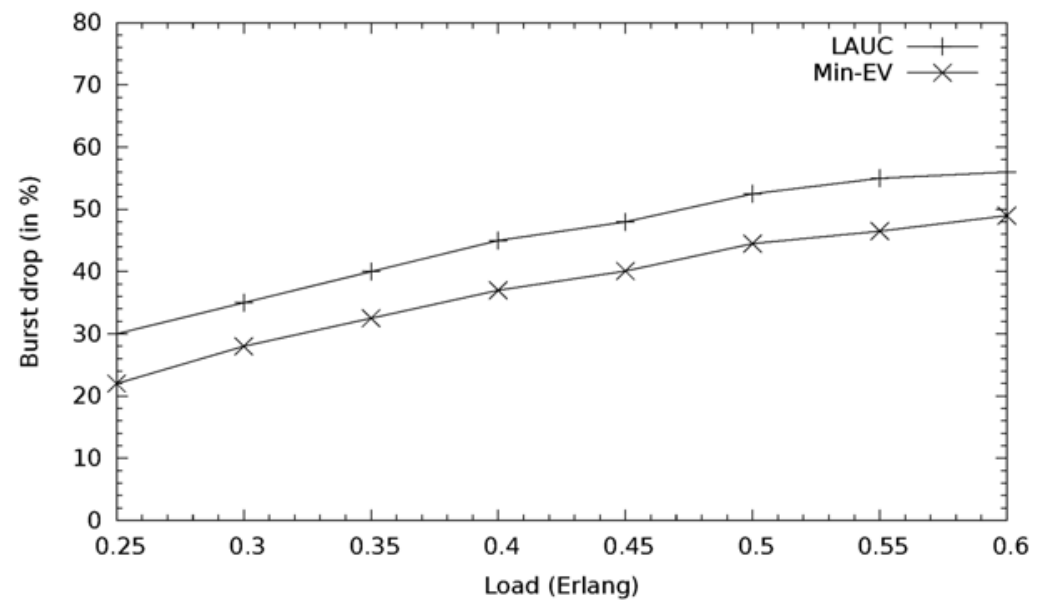

Fig. 4. Performance of algorithms under self-similar traffic.

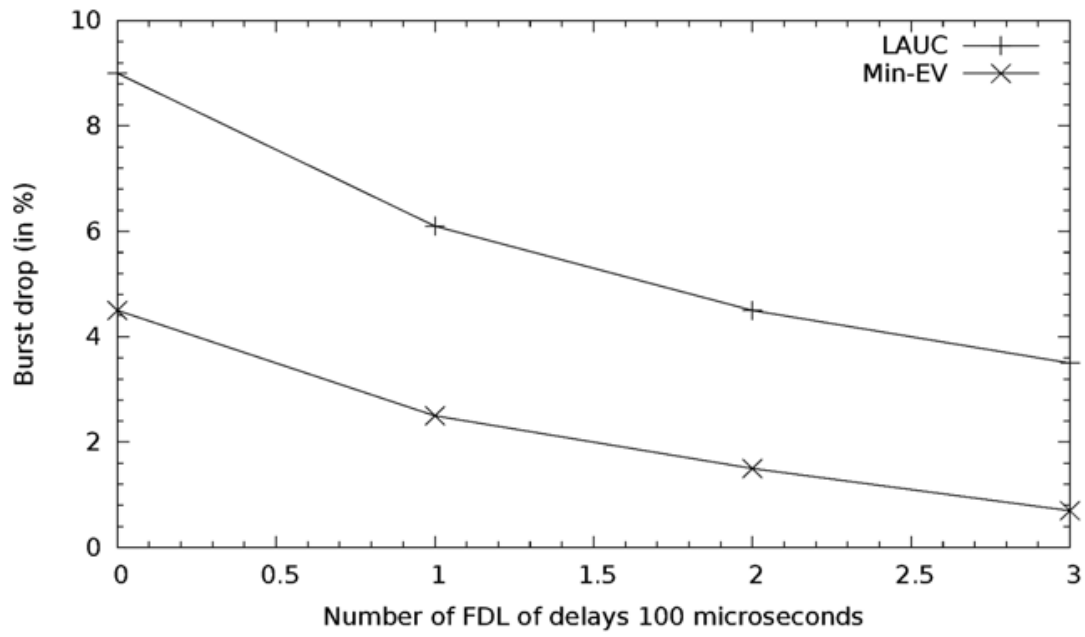

Fig. 5. Performance of LAUC algorithm under poisson traffic with and without FDLs; per outgoing channels.

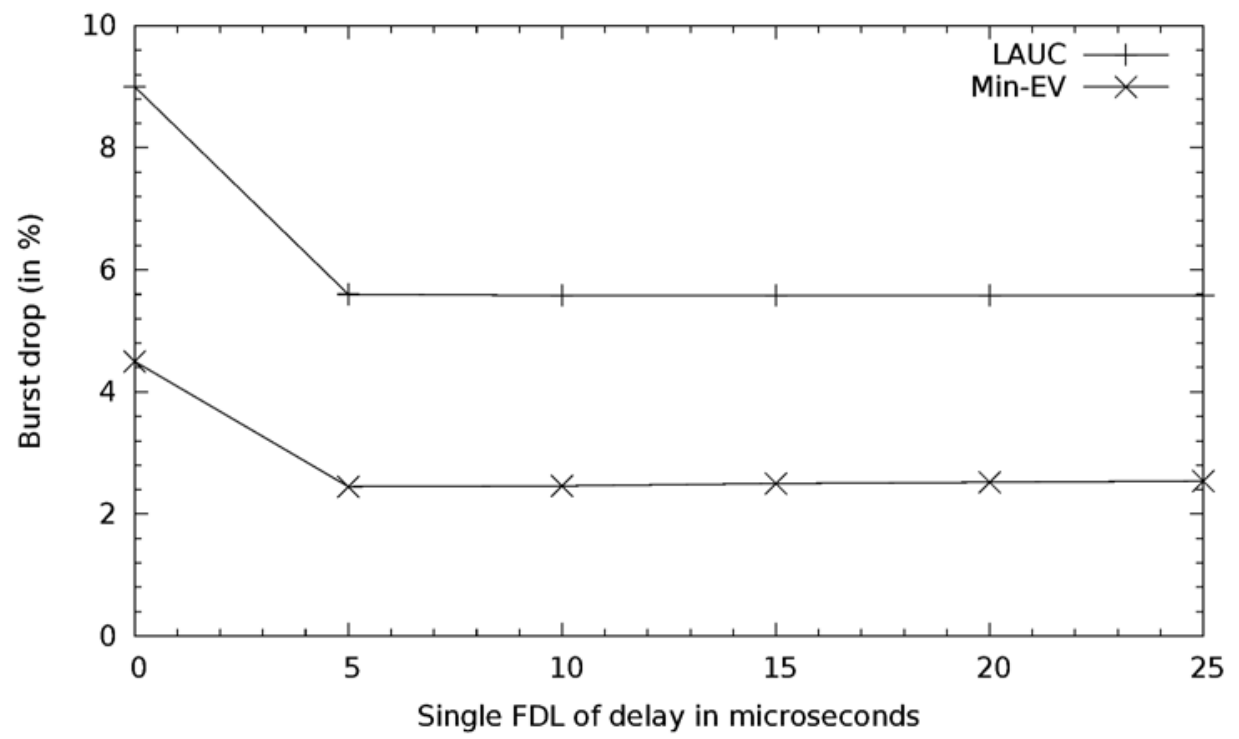

Fig. 6. Performance of class 1 traffic for various algorithms under Poisson traffic using optical FDL of delay in $\mu \mathrm{s}$. 
In order to evaluate the performance of OBS with burst dropping probability, we developed a simulation model. In our simulation, we consider the network topology which consists of 24 core and 16 edge nodes with 43 bidirectional links. Average node degree is 3.58 and average hop count $(\mathrm{H})$ is 2.99 . A bidirectional link is realized by two unidirectional links in the opposite direction. Each unidirectional link consists of 8 data channels and 1 control channel. Burst arrivals in the network are self-similar or poisson with arrival rate $\lambda$. Bursts are generated by using threshold-based assembly schemes and value of threshold is $40 \mathrm{~KB}$. Packet length is kept as 2000 bytes. Shortest path is used for routing the burst from source to destination. Two different classes of traffic are considered, namely class 1 (Lower priority) and class 2 (Higher priority). BHP processing time at each node $(\delta)$ is $1 \mu \mathrm{s}$.

Offset time of class 1 traffic is $\delta \mathrm{H}$. Offset time of class 2 traffic is $10 \delta \mathrm{H}$ which is greater than class 1 traffic. Range of traffic load is from 0.2 to 0.6 . Bursts are uniformly distributed over all sender-receiver pairs. Fig. 5, indicates that Min-EV(Void filling algorithm) has better performance in terms of burst dropping percentage than LAUC algorithm.

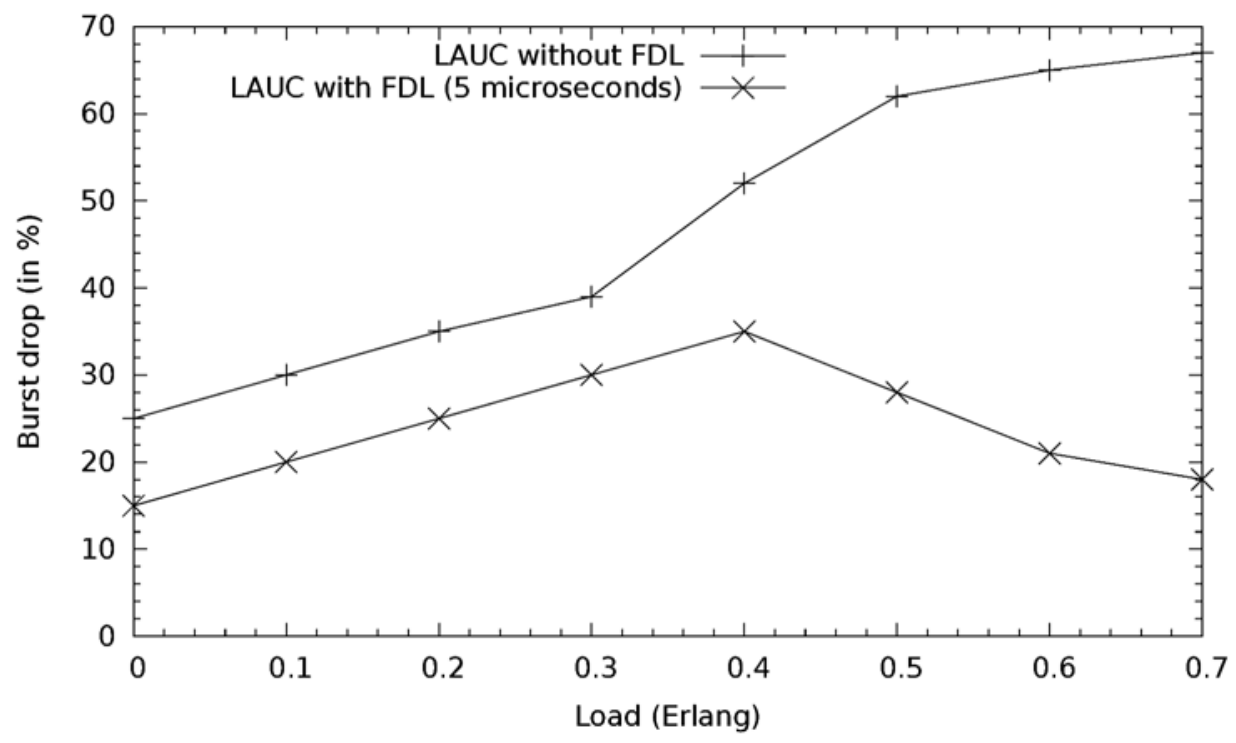

Fig. 7. Performance of LAUC algorithm under poisson traffic with and without FDLs.

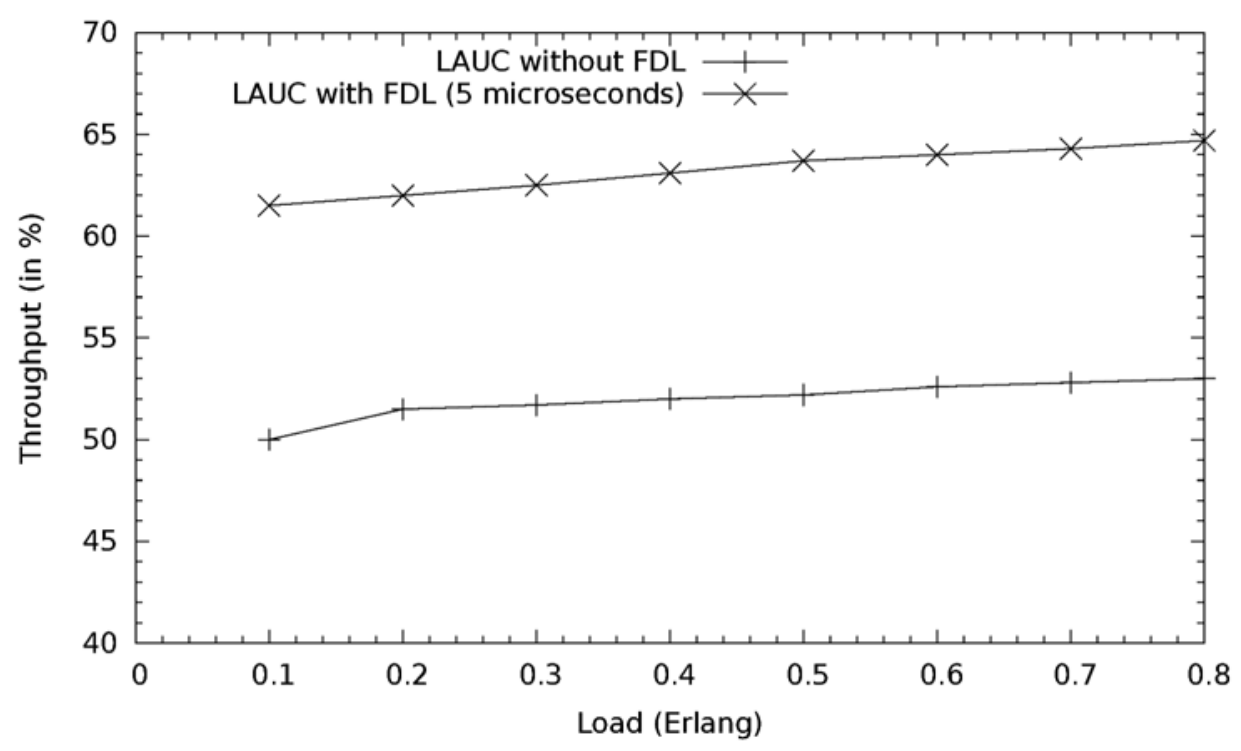

Fig. 8. Throughput of LAUC algorithms under poisson traffic with and without FDLs. 
Initially, the burst dropping percentage in OBS network for poisson traffic without FDLs is calculated then FDLs per outgoing channels are increased from zero to three; the class 1 traffic observations are shown in Fig. 5, for LAUC and Min-EV algorithms. It is observed that in both algorithms burst dropping percentage decreased as FDLs per out-going channels are increased. At the same time, it observed that the burst dropping percentage is less in case

of Min-EV algorithm. Instead of using FDLs per outgoing channels if we will use small fiber delay lines at the core nodes ranging from 5 to $25 \mu$ s one at a time as feed-forwarded buffer then we get the amazing results i.e we get less burst dropping percentage by using just one FDL of few $\mu$ s delay at the core node; which is contention resolution approach using optical buffering. Following Fig. 6, shows that we get the equivalent dropping percentage with $5 \mu$ s delay fiber, which is generally achieved by two long enough fibers per outgoing channel of hundreds of kilometers in length. We can delay burst by $1 \mathrm{~ms}$ using fiber of $200 \mathrm{~km}$ in length which is not cost effective approach. So, efficient way to reduce the burst loss percentage and to increase the throughput is contention resolution approach. Figs. 7, 8, 9 and 10 indicates that the burst dropping probability decreased and throughput increased as we used optical buffer at the core nodes.

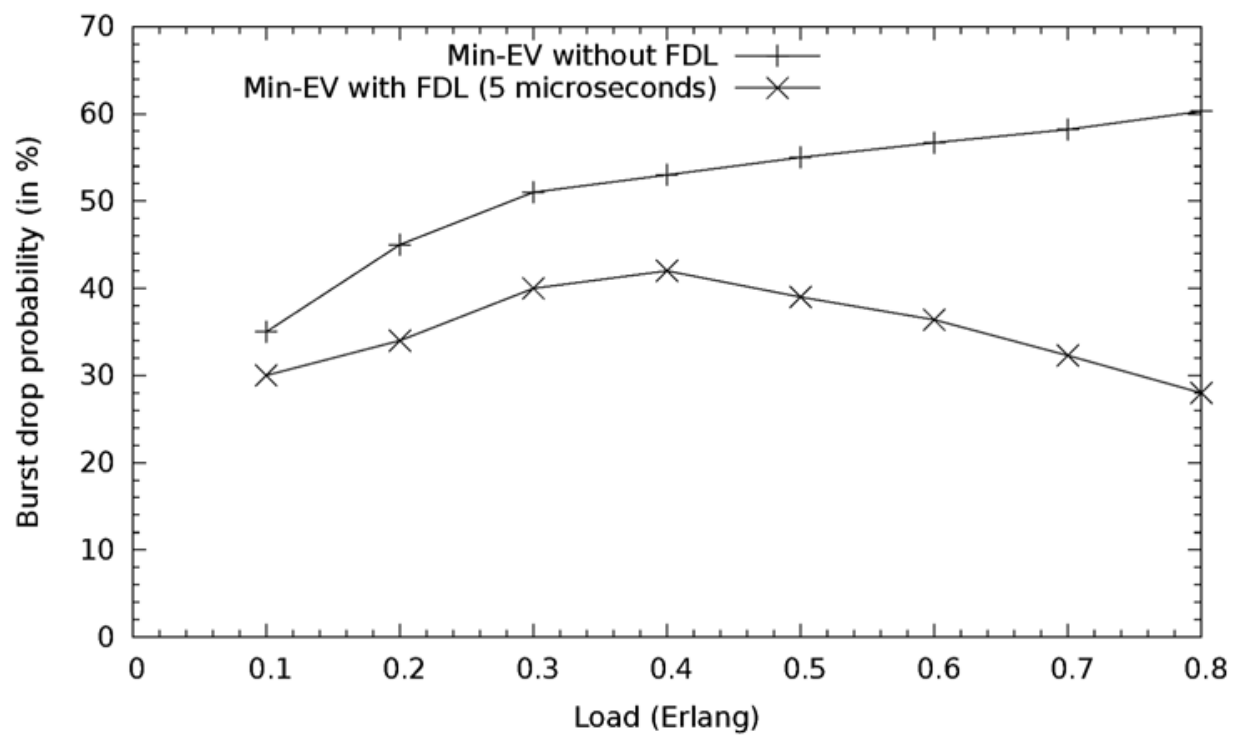

Fig. 9. Performance of Min-EV algorithms under poisson traffic with and without FDLs.

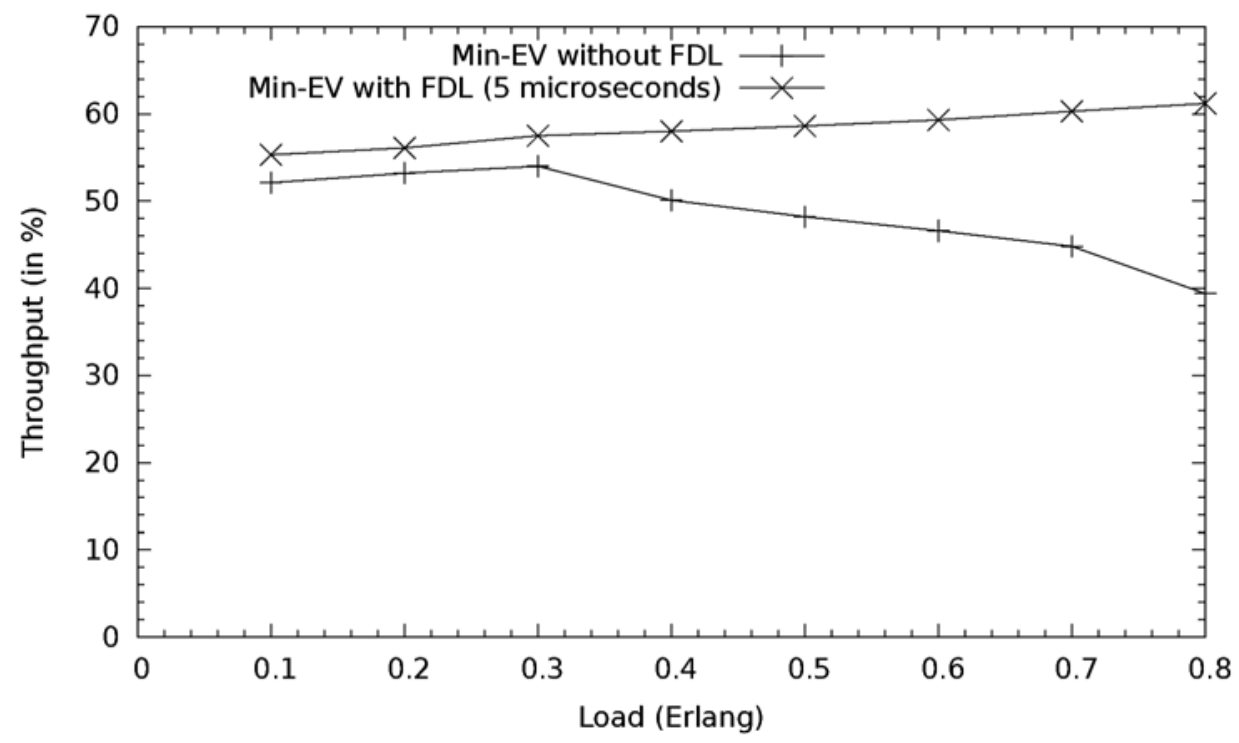

Fig. 10. Throughput of Min-EV algorithm under poisson traffic with and without FDLs. 


\section{Conclusion}

In this work, under the threshold burst assembly scheme with NSF network topology, we study the characteristics FDLs which affect burst dropping probability. The different traffic like self-similar and poisson is applied to OBS network. The performance of LAUC, and Min-EV is investigated through simulation. As threshold based scheme generate the same size of burst, all void filling algorithm like LAUC-VF, BF, Min-EV and Min-SV have the less burst dropping probability under self-similar and poisson traffic. It is also noticed that a threshold value increases the burst dropping probability decreases. It has been noticed that the self-similar process gives us the better results with large scale analysis and generate huge amount of traffic, but poisson process unable to provide it with the same condition. Burst dropping percentage can be minimized and throughput of the network can increase with the help of small fibers at a core node of delay in $\mu$ s instead of long fibers per outgoing channel of delay $100 \mu \mathrm{s}$. For self-similar traffic the similar trends of the graphs can be obtained. The ultimate aim is to increase the network throughput using optical buffers at the core node. Hence, Contention resolution with the help of single stage optical buffer; which is one of the parameters to improve the QoS of OBS network is verified. From the present results, our future work is to analyze the effect of the Hurst parameter under self-similar traffic as well as Poisson traffic for different types of optical buffers given in this paper with threshold based scheme. As self-similar traffic is justified traffic the results for this is analyzed in this paper.

\section{Acknowledgements}

The work described in this paper carried out with the financial support of University Grants Commission (UGC) through Minor Research Project (MRP). Further, this study is performed in the Department of Computer Engineering at Dr. BATU, Lonere Dist. Raigad, India. I would like to thank UGC MRP team for their financial support and our University for administrative and technical supports.

\section{References}

[1] B. Mukharjee, "Optical WDM Networks", Springer Publication, 2006.

[2] Tzvetelina Battestilli, "Optical Burst Switching: A Survey", Technical Report, NC State University, Computer Science Department, July 2002.

[3] C. Qiao and M. Yoo, "Optical Burst Switching: A New Paradigm For An Optical Internet", Journal of High Speed Network, Vol. 8, Pp. 69-84, 1999.

[4] Takuji Tachibana and Soji Kasahara, "Performance analysis of timer- based burst assembly with slotted scheduling for optical burst switching network", Performance Evaluation an International Journal, Vol. 63, Pp. 1016-1031, 2006.

[5] B. Praveen, J. Praveen and C. Siva Ram Murty, "A Survey of differentiated QoS schemes in optical burst switched networks", Optical Switching and Networking, Vol. 3, Pp. 134-142, July 2006.

[6] Noureddine Boudriga, "Optical burst switching protocols for supporting QoS and adaptive routing", Computer Communications, Vol. 26, Pp.1804-1812, 2003.

[7] Jason P. Jue and Vinod V. Vokkarane, "Optical Burst Switching Networks", Springer Publication, 2005.

[8] G. Mohan, K. Akash and M. Ashish, "Efficient techniques for improved QoS performance in WDM optical burst switched networks", Computer Communications, Vol. 28, Pp. 754-764, 2005.

[9] Konstantinos Chistodoullopoulos, Emmanouel Varvarigos and Kyriakos Vlachos, "A new burst assembly scheme based on average packet delay and its performance for TCP traffic", Optical Switching and Networking, Vol. 4, Pp. 200-212, 2007.

[10] Wang Ruyan, Wu Dapeng and Guo Fang, "Data Burst Statistics and Performance Analysis of Optical Burst Switching Networks with Self Similar Traffic", IEEE Computer Society, 2007.

[11] Jinhui Xu., Chunming Qiao, Jikai Li and Guang Xu, "Efficient Channel Scheduling Algorithms in Optical Switched Networks using Geometric Technique", IEEE Journal on selected areas in Communication, Vol. 22, No. 9, November 2004.

[12] Jikai Li and Chunming Qiao, "Schedule burst proactively for optical burst switching networks", Computer Networks, Vol. 44, Pp. 617-629, 2004.

[13] Burak Kantarci, Sema F. Oktug and Tulin Atmaca, "Performance of OBS techniques under self-similar traffic based on various burst assembly techniques", Computer Communications, Vol. 30, Pp. 315-325, 2007.

[14] S. K. Tan, G. Mohan and K. K. Chua, "Burst Rescheduling with Wavelength and Last-hop FDL Reassignment in WDM Optical Burst Switching Networks”, IEEE Computer Networks, 2003. 